\title{
The three-dimensional architecture of the internal capsule of the human brain demonstrated by fiber dissection technique
}

1. University of Medicine and Pharmacy Tirgu Mures, Tirgu Mures, Romania, Department of Anatomy

2. Cluj County Emergency Hospital, Cluj Napoca, Romania, Department of Neurosurgery

3. Yeditepe University School of Medicine, Istanbul, Turkey, Department of Neurosurgery

\begin{abstract}
The fiber dissection technique involves peeling away white matter fiber tracts of the brain to display its three-dimensional anatomic arrangement. The intricate three-dimensional configuration and structure of the internal capsule (IC) is not well defined. By using the fiber dissection technique, our aim was to expose and study the IC to achieve a clearer conception of its configuration and relationships with neighboring white matter fibers and central nuclei.

The lateral and medial aspects of the temporal lobes of twenty, previously frozen, formalin-fixed human brains were dissected under the operating microscope using the fiber dissection technique.

The details of the three-dimensional arrangement of the fibers within the IC were studied and a comprehensive understanding of their relations was achieved. The white matter fiber dissection provides an enhanced perspective of the intricate architecture of the internal structure of brain. This enhanced understanding of intrinsic brain anatomy, particularly of functional highly relevant fiber systems such as the internal capsule, is essential for performing modern neurosurgical procedures.
\end{abstract}

Keywords: internal capsule, fiber dissection technique, white matter anatomy, microsurgical anatomy

Klara Brinzaniuc, M.D., Ph. D.

Department of Anatomy, University of Medicine and Pharmacy Tirgu Mures

Gheorghe Marinescu Street, No. 38, Tirgu Mures, Mures, 540139, Romania

Telephone: +40-265-21 55 51/163 / Fax: $\quad$ +40-265-21 0407

Mobile: +40-745-66.78.11; +40-732-50.01.61

E-mail: klara_branzaniuc@yahoo.com

\section{Introduction}

The white matter of the brain consists of bundles of myelinated nerve fibers known as fascicles or fiber tracts. There are three groups of nerve fibers: association, connection and projection fibers. Association fibers connect neighboring and distal cortical region in the same hemisphere. Commissural fibers connect homologues regions in the two hemispheres. Projection fibers connect the cerebral cortex with subcortical structures such as thalamus, basal ganglia, brainstem and spinal cord. These projection fibers radiate to and from the entire cortical surface to pass through the corona radiata and continue in a compact arrangement within the IC. The IC is the term applied to the broad white matter band, which intervenes between the lenticular nucleus, putamen and globus pallidus, on the lateral side and the caudate nucleus and the thalamus on the medial side [1-3].

The fiber dissection technique involves peeling away the white matter tracts to display its internal anatomical organization. This technique reveals the intricate three-dimensional organization and relationship among the association, commissural and projection fibers of the brain. The details of the intricate three-dimensional configuration of projection fibers within the IC continue to elude us. Standard depictions of the IC are mostly derived from serial 
sections of the brain or from schematic diagrams. However, is very difficult to reconstruct, from the study of two-dimensional serial sections of the brain, an accurate and complete mental representation of complex fiber systems, such as the IC. The threedimensional complex structure, configuration and relations of the IC might be particularly revealed with the fiber dissection technique. This report aims to reconsider the three-dimensional anatomy of the IC as well as to provide a clear conception about its configuration and spatial relations by using the fiber dissection technique.

\section{Material and methods}

We dissected 20 previously frozen, formalinfixed human brains under the operating microscope using the fiber dissection technique [4,5]. The brains were removed from craniums no later than 10-12 hours postmortem. To prevent alteration of the normal contour of the brain, the basilar artery was ligated and used to suspend the brains in $10 \%$ formaldehyde solution for at least 2 months. The arachnoid membranes were removed and the brain specimens were then washed in running water for several hours to remove the formalin and refrigerated at temperatures ranging between -10 and $-15 \mathrm{C}$ for 1 week. Afterwards they were immersed in water and allow to thaw. Arachnoid membranes were then removed and specimens dissected using the operative microscope with X6-X40 magnification. The dissection was performed using fine watchmaker forceps, wooden spatulas with various tip sizes and delicate microtechniques. A step-wise dissection of the lateral and medial aspects of the cerebral hemisphere was performed, as previously recommended by Ture [6-8].

\section{Results}

The dissection of the lateral aspect of the cerebral hemisphere proceeds as follows (Figure 1A). After identification of the sulcal and gyral patterns on the lateral aspect of the brain the frontoorbital, frontoparietal and temporal operculum are elevated to expose the insula surrounded by the superior longitudinal fasciculus (Figure 1B). The superior longitudinal fasciculus is a $\mathrm{C}$-shaped, long association fiber system that connects frontal, parietal, occipital and temporal regions and is located in depth of middle frontal gyrus, inferior parietal lobule and middle temporal gyrus.

The insula is an invaginated portion of cerebral cortex that forms the base of the sylvian fissure. The insular cortex and the underlying extreme capsule, claustrum and external capsule are dissected away to reveal the putamen, with the corona radiata at its periphery (Figure 1C). The occipitofrontal fasciculus is a long association fiber system located underneath limen insula that connects the frontal and occipital lobes. The occipitofrontal fasciculus is demonstrated at the base of the putamen, passing in the layer of the extreme and external capsules and joining the sagittal stratum.

The sagittal stratum is a longitudinal fiber system in the temporal and occipital lobes, that is fully revealed by further dissecting the inferior temporal gyrus and fusiform gyrus. A portion of the sagittal stratum stands for the equivalent of the corona radiata in the temporal and occipital regions. Corona radiata is a projection fiber system that continues the IC in the frontal and parietal regions, whereas the sagittal stratum contains, in addition to IC fibers for the temporal and occipital regions, the occipitofronatal fasciculus and anterior commissure fibers

The putamen is, next, removed to reveal the globus pallidus and the IC at its periphery (Figure 1D). The globus pallidus has a firmer consistency, enabling the differentiation from the softer, spongy putamen. The strionigral fibers that pass through the globus pallidus can be identified with higher magnification. The caudolenticular grey matter that 



Figure 1 - Photographs of brain specimens showing serial dissections of lateral aspect of the left cerebral hemisphere.

A: Lateral aspect of the left cerebral hemisphere. B: Dissecting the frontoparietal, frontoorbital and temporal operculum reveals the superior longitudinal fasciculus (slf) surrounding the insula. C: Removal of the insular cortex, extreme capsule, claustrum, and external capsule demonstrates the occipitofrontal fasciculus (off), putamen (pu) and corona radiata (cr). Extensive dissection of the basal temporal lobe demonstrates the sagittal stratum (sas). D: After the putamen is removed, the globus pallidus (gp) and the internal capsule at its periphery are seen. The lateral extension of the anterior commissure (ac) into the sagittal stratum (sas) is revealed underneath the occipitofrontal fasciculus. E: The lateral extension of the anterior commissure is severed and the globus pallidus is removed to fully expose the lateral aspect of the anterior limb (ic-a), posterior limb (ic-p) retrolentiform (ic-rl) and sublentiform portions of the internal capsule (ic-sl). F: Removing the amygdaloid body $(a b)$ and severing of the anterior commissure demonstrates the continuation of the internal capsule fibers into the cerebral peduncle of midbrain (m-cp) lateral aspect of the sublentiform portion of the internal capsule. Abbreviations with white letters denote the sulci and fissures. Ang = 
angular gyrus; cer = cerebellum; $c s=$ central sulcus of Rolando; $F 1=$ superior frontal gyrus; $F 2=$ middle frontal gyrus; F3 = inferior frontal gyrus; $f 1=$ superior frontal sulcus; $f 2=$ inferior frontal sulcus; $i-a=$ insular apex; $i-l$

$=$ limen insula; $i c-a=$ anterior limb of the internal capsule; $i c-p=$ posterior limb of the internal capsule; ic- $r l=$ retrolentiform portion of the internal capsule; $n a=$ nucleus accumbens; $\mathrm{O} 1=$ superior occipital gyrus; $\mathrm{O} 2=$ middle occipital gyrus; $\mathrm{O} 3$ = inferior occipital gyrus; op = pars opercularis of F3; or = pars orbitalis of F3; po = pons; pog = postcentral gyrus; pos = postcentral sulcus; prg = precentral gyrus; prs $=$ precentral sulcus; $p s-a=$ anterior periinsular sulcus; $p s-i=$ inferior periinsular sulcus; $p u=$ putamen; $s f=$ sylvian fissure; $s m g=$ supramarginal gyrus; $s p l=$ superior parietal lobule; $T 1=$ superior temporal gyrus; $t 1=$ superior temporal sulcus; $T 2=$ middle temporal gyrus; $t 2=$ inferior temporal sulcus; $T 3=$ inferior temporal gyrus; $t p=$ temporal pole; $t r=$ pars triangularis of $F 3$.

passes through the IC and connects the caudate and putamen can also be identified.

The anterior commissure is revealed at the base of the globus pallidus and connects homologues olfactory, temporal and occipital regions. The compact cylindrical structure in the midline gives off a small component of fibers, the anterior extension (anterior limb), that join the IC and reach olfactory regions in the basal frontal lobe. Most of the fibers of the compact anterior commissure continue laterally (lateral extension, posterior limb), at the base of the globus pallidus, to overlap and intermingle with the occipitofrontal fasciculus within the sagittal stratum.

Next, the occipitofrontal fasciculus is dissected away, the lateral extensions of the anterior commissure are followed into the sagittal stratum and severed and globus pallidus is removed to completely expose the lateral aspect of the IC (Figure 2E). The lateral aspect of the IC presents an evenly concave surface within which the lentiform nucleus is received. The sharp bend, corresponding to the genu, cannot be precisely identified on this side of the IC.

Based on the locations and orientation patterns of the fibers, 2 components of the IC could be clearly identified: the anterior limb (lenticulocaudate portion), between the lentiform nucleus and caudate nucleus and posterior limb (lenticulothalamic portion), between the lenticular nucleus and thalamus. The posterior limb is more complex and might be subdivided into: lenticulothalamic, retrolentiform and sublentiform portions (Table I). The corticopontine and corticospinal fibers are the components of the IC revealed on the lateral aspect of the IC, whereas the thalamocortical and corticothalamic fibers - thalamic peduncles - are revealed on the medial aspect.

The three-dimensional arrangement of these

Table I - Internal capsule configuration

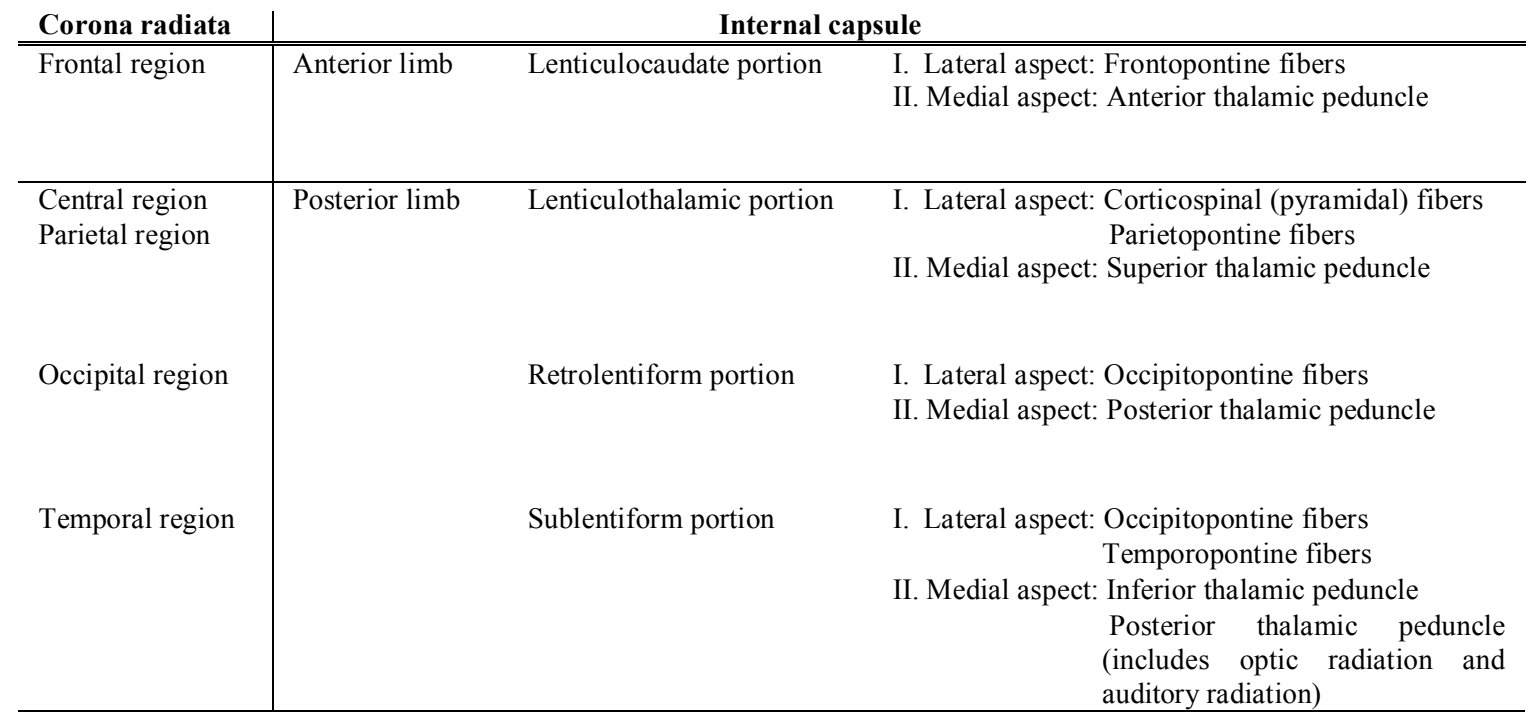




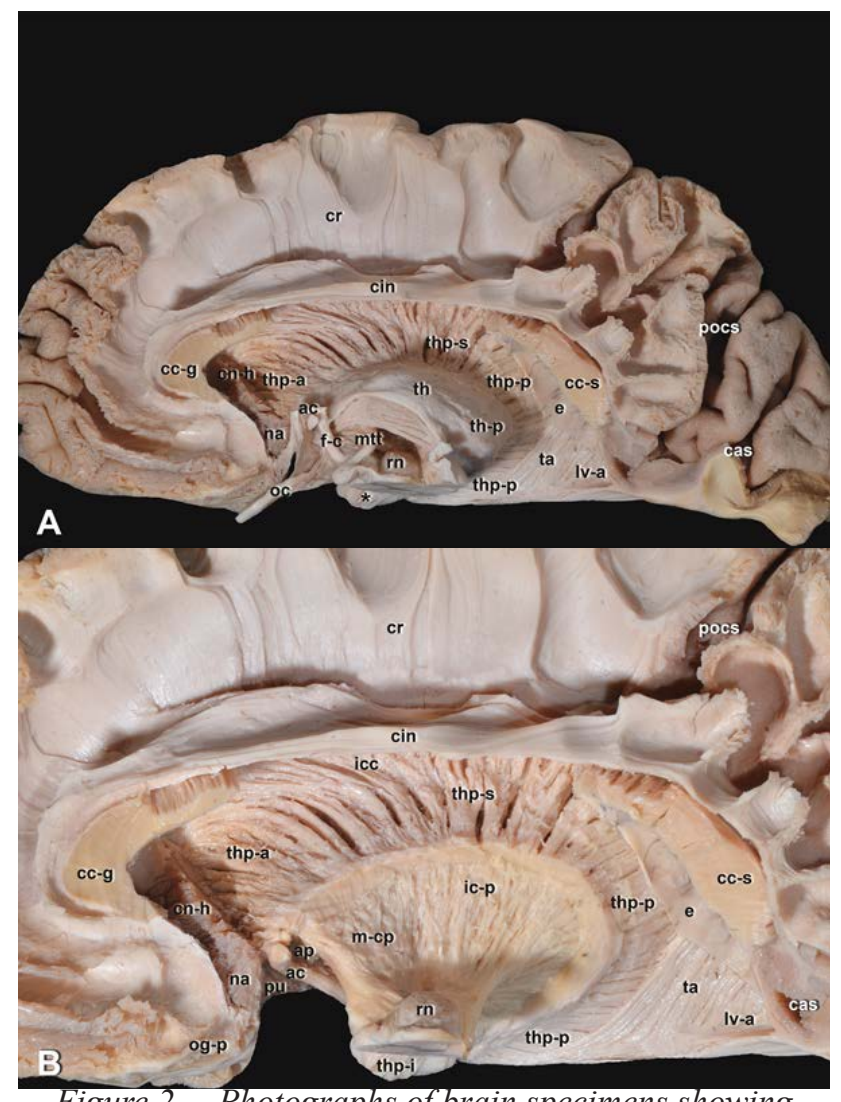

Figure 2 - Photographs of brain specimens showing serial dissections of medial aspect of the left cerebral hemisphere. A: Extensive dissection of the medial aspect of the brain hemisphere to demonstrate the cingulum (cin) and corona radiata (cr). Removing the ependima of the lateral walls of the third ventricles and partial removal of the caudate nucleus reveal the anterior thalamic peduncle (thp-a), superior thalamic peduncle (thp-s), posterior thalamic peduncle (thp-p) and inferior thalamic peduncles (thp-i) and tapetum (ta). Dissecting the lateral third ventricular wall demonstrates the mamillothalamic tract (mtt), columns of fornix $(f-c)$ and superior portion of the red nucleus (rn). B: Removal of the thalamus fully reveals the medial aspect of the internal capsule extending upward into the corona radiata (cr) and downwards to the cerebral peduncle of midbrain (m-cp). Abbreviations with white letters denote the sulci and fissures. Ac = anterior commissure; ap = ansa peduncularis; cas = calcarine sulcus; $c c-b=b o d y$ of the corpus callosum; cc-s = splenium of the corpus callosum; cos = collateral sulcus; II = optic nerve; lv-a = atrium of the lateral ventricle; $m g b=$ medial geniculate body; og- $p=$ posterior orbital gyri; ot = optic tract; pocs = parietoocipital sulcus sulcus; pon= preoccipital notch; th $=$ thalamus components of the IC was evident in our dissections. The fibers of the anterior limb and the lenticulothalamic portion of the posterior limb course forward or upward in an almost vertical, sagittal plane to continue in the corona radiata and intermingle with the fibers of the corpus callosum; the fibers of the retrolentiform portion course in a almost coronar plane, behind the lentiform nucleus, and then turn backwards to continue within the sagittal stratum; whereas, the fibers of the sublentiform portion of the IC run in an axial, horizontal plane beneath the lentiform nucleus to interwoven with the occipitofrontal fasciculus and anterior commissure and form the sagittal stratum.

Within the sagittal stratum, the sublentiform portion of the IC displays a detour into the temporal lobe - a loop of the fibers into the anterior temporal region, the temporal loop or Meyer's loop - before turning backwards and continuing towards the temporal and occipital cortical regions. Furthermore, within the sagittal stratum, it was not possible to differentiate distinct component fibers.

By further severing the lateral extension of the anterior commissure and underlying ansa peduncularis and removing the amygdaloid body, the continuation of the IC fibers into the cerebral peduncle is fully revealed (Figure $1 \mathrm{~F}$ ). The frontopontine fibers, of the anterior limb of IC, arch downwards, over the anterior commissure and pass into the medial fifth of the cerebral peduncle. The corticospinal fibers (pyramidal tract) of the lenticulothalamic portions and parietopontine fibers of the retrolentiform portion of the IC pass downwards, behind the optic tract and into the cerebral peduncle. The sublentiform portion of the IC fibers - the occipitopontine and temporopontine fibers - passes over the optic tract and arch downwards to reach the lateral fifth of the cerebral peduncle.

An instructive view of the IC can be obtained by dissecting the medial aspect of the cerebral hemisphere (Figure 2A). Extensive dissection of corpus callosum, cingulate gyrus and base of the temporal lobe reveals the entire anatomy of the lateral and third ventricles.

Dissecting away the caudate nucleus and thalamus demonstrates the medial aspect of the IC (Figure 2B). Both these structures make a distinct impression on the medial aspect of the IC and 
between these structures there is a prominent curved ridge. Beyond this ridge, corresponding to the stria terminalis, the thalamocortical and corticothalamic fibers of the anterior, superior, posterior and inferior thalamic peduncles are demonstrated. The thalamic peduncle fibers parallel the orientation patterns of the, laterally placed, corticopontine and corticospinal fibers. Below this ridge, the thalamic impression on the lenticulothalamic and retrolentiform portions of the IC creates a sharp dihedral angle between the anterior and the posterior limbs of the IC. This thalamic impression is markedly concave and belongs entirely to posterior thalamic peduncle.

\section{Discussion}

The long and eminent history of the observations that lead to the present understanding of the anatomy and function of the internal capsule begins in the 16th century with Vesalius, who rendered the first illustration [9]. Dissection of the white matter fibers was used, for the first time by Vieussens, in 1685, to describe and name the centrum semiovale and demonstrate the continuity with the internal capsule, cerebral peduncles and pyramidal tracts in the pons and medulla oblongata [10]. In 1807, Reil emphasized these anatomical relationships, and introduced the term "corona radiata" for the radiating fibers of the centrum semiovale [11]. Gall and Spurtzheim dissected the internal capsule and the centrum semiovale [12]. Mayo published exquisite depiction of the internal capsule [13], while Arnold illustrated these relationships in detail in his superb atlas published between 1838-1840 [14]. Other early anatomist demonstrated many fiber systems using the fiber dissection technique $[15,16]$.

With the advent of the microtome and the development of histological coloration methods, the histological techniques became subsequently preferred [17-19]. With the histological techniques, it is very difficult to follow white matter fiber tracts for long distances. Understanding the three-dimensional configuration of the internal capsule from the study of serial sections, is particularly difficult, because of the intricate arrangement of the neighboring fiber systems and complex relations with the basal ganglia and diencephalic nuclei, and for this reason techniques that might be able to display the true spatial form and relations, such as the fiber dissection, might be particularly instructive.

Few anatomists during the early 20th century, such as Johnston, Hoeve and Curran advocated the fiber dissection technique for studying the complex relationships of the white matter fiber systems [2022]. A comprehensive description and illustration of the internal capsule was provided by Ranson, in 1921 [23]. In 1935, Klingler developed an improved method of brain fixation and fiber dissection that came to be known as "Klingler fiber dissection technique ". The special freezing and dissection technique developed by Joseph Klingler, allowed for a new level of anatomical accuracy in the white matter investigations $[4,5,24]$. He elaborated impressive preparations, using this technique of dissection and produced a comprehensive atlas, rendering his meticulous and elegant dissections.

Recently, Ture et al. revitalized interest in the fiber dissection technique and strengthen its essential role for enhancing neurosurgical procedures [6-8]. By further refining the fiber dissection technique and addition of the operating microscope to study the detail of the fiber systems, this method proved to be especially relevant for an accurate threedimensional perspective of the brain complex structure and augmentation of our knowledge about the brain anatomical features. This increased knowledge of the brain anatomical features is invaluable for neurosurgeons, neuroradiologists, neurologists, neuropsychiatrist, neurophysiologists and neuropathologists.

The projection fibers, that encompass a major portion of the fibers that emanate from each cortical area, form distinct fiber systems destined for the thalamus, nuclei of the basis pontis, other diencephalic and brainstem structures and the spinal cord. The internal capsule refers to the compact portion of white matter projection fibers interposed between the basal ganglia and diencephalus. Its component fibers reach their cortical terminations by diverging in a radiate 
pattern -forward, upward, backward and lateralward to form the corona radiata. These fibers continue their course downwards and are compacted even further within the crus cerebri of the mesencephalus.

The shape of the internal capsule is variable according to the plane of examination. In coronar sections through the cerebral peduncle, the internal capsule appears as a vertical band of white matter, in direct continuation with the basal part of the cerebral peduncle. The standard "V-shape" configuration, with an anterior limb, genu and posterior limb, appears only in axial brain sections.

In our lateral and medial fiber dissection preparations, the three-dimensional shape of the white matter compact layer, molded by the basal ganglia and thalamic nuclear masses, was particularly evident. The lateral aspect of the internal capsule displays an evenly concave surface within which the lentiform nucleus is received. There is no sharp bend in the surface to correspond to the genu. On the medial aspect of the internal capsule, each of the nuclear masses, caudate nucleus and thalamus, make a distinct impression on the medial surface of the capsule. Between these 2 concave, impression there is a sharp dihedral angle. This dihedral angle follows the line of the sulcus which separates the caudate nucleus from thalamus and which contains the stria terminalis. This dihedral angle represents the sharp genu in axial sections of the brain only, at some appropriate levels [23].

The caudate nucleus impression corresponds in outline to the shape of the caudate nucleus: a large impression corresponding to the head and a narrower impression as, the caudate nucleus tappers. The thalamic impression is markedly concave and corresponds only to the posterior limb of IC.

\section{Conclusions}

Dissecting the fiber tracts of the white matter attains an enhanced understanding and more accurate knowledge of the three-dimensional internal configuration of the brain. This knowledge is invaluable for performing modern neurosurgical procedures for epilepsy or intrinsic brain tumors, arteriovenous malformations, cavernous malformations. Acquiring experience and proficiency with this technique is recommended.

\section{Acknowledgements}

We are grateful to Professor Uğur Türe for support and guidance during completion of the study at the Microsurgical Anatomy Laboratory of the Department of Neurosurgery at the Yeditepe University School of Medicine in Istanbul.

This paper was partly supported by the Sectorial Operational Programme Human Resources Development (SOPHRD), financed from the European Social Fund and by the Romanian Government under the contract number POSDRU 80641.

\section{References}

1. Cunningham, D.J. \& Robinson, A. (1918). Cunningham's Textbook of Anatomy (5th ed.). New York William: Wood and Company

2. Carpenter, M.B. \& Sutin, J. (1983). Human Neuroanatomy (8th ed.) Baltimore, ML: Williams \& Wilkins

3. Standring, S. (2008). Gray's Anatomy (40th ed.). New York: Elsevier Churchill Livingstone

4. Klingler, J. \& Gloor, P. (1960). The connections of the amygdala and of the anterior temporal cortex in the human brain. Journal of Comparative Neurology. 115, 333-369.

5. Ludwig, E. \& Klingler, J. (1956). Atlas Cerebri Humani. Basel: S Karger

6. Türe, U., Yaşargil, M.G. \& Friedman, A.H. 
(2000). Fiber dissection technique: lateral aspect of the brain. Neurosurgery. 47, 417-426

7. Türe, U., Yaşargil, M.G. \& Pait, T.G. (1997). Is there a superior occipitofrontal fasciculus? A microsurgical anatomic study. Neurosurgery. 40, 1226-1232

8. Yaşargil, M.G., Türe, U. \& Yasargil, D.C. (2005). Surgical anatomy of supratentorial midline lesions. Neurosurg. FOCUS. 18(E6), E1-E1.

9. Vesalius, A. (1543). The humani corporis fabrica. Basil: Johann Oporinus

10. Vieussens, R. (1685). Neurographia Universalis. Lyons: Lugundi, Apud Joannem Certe,

11. Reil, J.C. (1807). Fregmente über die bildung des kleinen gehirns im menschen. Arch. Physiol. Halle. 8, 1-58.

12. Gall, F.J. \& Spurzheim, J.C .(1810). Anatomie et Physiologie du Systeme Nerveux en General et $d u$ Cerveau en Particulier. Paris F: Schoell

13. Mayo, H.M. (1827). A Series of Engravings Intended to Illustrate the Structure of the Brain and Spinal Cord in Man. London: Burgess Hill

14. Arnold, F. (1838). Tabulae Anatomicae: Icones Cerebri et Medullae Spinalis. Turici: Orelli, Fuesslin,

15. Gratiolet, P.L. (1854). Mémoires sur les Plis Cérébraux des Primates. Paris: Bertrand
16. Burdach, K.F. (1844). Umrisse einer Physiologie des Nervensystems. Leipzig: Lopold Boss

17. Dejerine, J.J. (1895). Anatomie des Centres Nerveux. J Paris: Rueff et Cie,

18. Flechsig, P.E. (1896). Weitere mitteilungen über den stabkranz des menschlichen grosshirns. Neurologishes. Centralblatt.15, 2-4

19. Flechsig, P.E. (1920). Anatomie des Menschlichen Gehirns und Rückenmarks auf Myelogenetischer Grundlage. Leipzig, Thieme

20. Johnston, J.B. (1908). A new method of brain dissection. Anat. Rec. 2, 345-358

21. Hoeve, H. (1909). A modern method of teaching the anatomy of the brain. Anat. Rec. 3, 247-257

22. Curran, E.J. (1909). A new association fiber tract in the cerebrum with remarks on the fiber tract dissection method of studying the brain. $J$. Comp. Neurol. 19, 645-656

23. Ranson, S.W. (1921). A description of some dissections of the internal capsule, the corona radiata and the thalamic radiation to the temporal lobe. Archives of Neurology and Psychiatry. 5(4), 361-369

24. Klingler, J. (1935). Erleichterung der Makroskopischen Präparation des Gehirns durch den Gefrierprozess. Schweiz. Arch. Neurol. Psychiat. 36, 247-256 